\title{
A Meta-Analysis of Randomized and Observational Studies: Aspirin Protects from Cardiac Surgery-Associated Acute Kidney Injury
}

\author{
Huijiao Liu, ${ }^{1}$ Jianwei Li, ${ }^{1}$ Miaolian Chen, ${ }^{1}$ Ting Yang, ${ }^{1}$ Zongfa Ruan, ${ }^{1}$ Jiahao Su, ${ }^{2}$ Yichun Xing ${ }^{3}$ \\ ${ }^{1}$ Department of Department of Critical Care Medicine, Zhongshan City People's Hospital, Zhongshan City, 528403 China; \\ ${ }^{2}$ Department of Neurosurgery, Zhongshan City People's Hospital, Zhongshan City, Guangdong Province 528403 China; \\ ${ }^{3}$ Department of Gynecology, Sun Yat-sen Memorial Hospital, Guangzhou, 5105120 China
}

\section{ABSTRACT}

Background: Antiplatelet therapy is critical in the management of coronary artery diseases. For patients undergoing cardiac surgeries, including coronary artery bypass graft $(\mathrm{CABG})$ and valve replacement, controversy remains in preoperative antiplatelet therapy concerning risk of bleeding. For safety concern, aspirin is recommended to be withdrawn 5 to 10 days before a cardiac surgery. Recent studies, however, indicate that preoperative aspirin may have a protective effect on cardiac surgery-associated acute kidney injury (CSA-AKI).

Objective: To estimate the efficacy of preoperative aspirin in preventing CSA-AKI.

Methods and results: Eligible studies included randomized controlled trials (RCTs) and observational studies (OSs) of patients, who had undergone CABG, valve replacement, or combined surgery. These studies compared preoperative aspirin with placebo/no aspirin and reported the least incidence of CSA-AKI. One RCT and five OSs met the inclusion criteria. Data retrieved suggested that aspirin prescribed within five days before cardiac surgery decreased post-operative renal failure [odds ratio (OR), 0.67; 95\% confidence interval (CI), 0.50-0.89; $\mathrm{P}<0.01]$ and 30-day mortality (OR, 0.64; $95 \% \mathrm{CI}$, $0.53-0.77 ; \mathrm{P}<0.01)$. One RCT and three OSs suggested aspirin protected from major adverse cardiocerebral events (MACE) (OR, 0.88; 95\% CI, 0.76-1.01; P = 0.07). One RCT and two OSs suggested aspirin did not increase risk of re-exploration for bleeding (OR, 1.01; 95\% CI, 0.76-1.34; $\mathrm{P}=0.95$ ).

Conclusion: Preoperative low-dose aspirin decreases postoperative CSA-AKI, mortality, and MACE without increasing the risk of re-exploration. But most of the studies are observational. They lack a uniformed standard on prescription of aspirin and outcomes measurement. No stratification analysis is performed concerning different types of surgical procedures and comorbidities. More randomized controlled trials are necessary to confirm the efficacy and safety of preoperative aspirin prescription.

Received February 2, 2019; received in revised form fune 19, 2019; accepted fune 20, 2019.

Correspondence: Huijiao Liu, Department of Department of Critical Care Medicine, Zhongshan City People's Hospital, Zhongshan City, 528403 China; (e-mail:15013228659@163.com).

\section{INTRODUCTION}

An estimated two million cardiac surgeries are performed annually worldwide [Rosner 2006], and nearly 400,000 cases of coronary artery bypass graft surgeries (CABG) proceed in United States every year as the first choice of complicated coronary artery disease (CAD) [Kulik 2015]. Acute kidney injury (AKI) is a critical complication of major cardiac surgeries with an incidence ranging from $2 \%$ to $40 \%$ (an average of $22 \%$ ) by different AKI definition or criteria [Fuhrman 2017; Hoste 2017]. Dialysis is required in approximately $1 \%$ of all patients [Rosner 2006]. Cardiac surgery-associated AKI (CSA-AKI) significantly increases in-hospital mortality almost tenfold and mean total index hospitalization costs [Hoste 2017; Alshaikh 2017]. It is estimated that a reduction of $10 \%$ AKIs in cardiac surgeries could save approximately $\$ 100$ million index-hospital costs annually [Alshaikh 2017].

The occurrence of CSA-AKI is physiologically explained by hemolysis and systemic inflammation reaction during extracorporeal circulation, vasoconstriction caused by neurohormonal factors and a low-flow, low-pressure, non-pulsatile perfusion [Fuhrman 2017; Wang 2017]. Vasodilatory shock due to post-cardiopulmonary-bypass vasoplegia also plays a role. All of the above factors eventually lead to ischemic-reperfusion injury. Current key principles for prevention of CSA-AKI are based on inductive physiological and phar $\neg$ macological thinking. They include restoring cardiac output by volume expansion, vasopressors and inotropic drugs administration, discontinuation of nephrotoxic agents, and glycemic control [Wang 2017]. Anti-inflammatory strategies to moderate inflammatory response, including glucocorticoid, miniaturized extracorporeal circuit, and the leukocyte filter, have been investigated [Shin 2016]. Recent observational studies also indicate that preoperative aspirin may have a protective effect on CSA-AKI [Hur 2017; Mazzeffi 2016; Huang 2015; Yao 2015; Cao 2012; Cao 2012].

Aspirin, or acetylsalicylic acid, is a classic nonsteroidal anti-inflammatory drug. It irreversibly inhibits activity of cyclooxygenase-1(COX-1), prevents formation of platelet thromboxane A2 (TXA2), and thus inhibits aggregation and activation of platelets [Fuster 1993]. It widely is used in CAD patients for its antiplatelet and antithrombotic effect. For patients undergoing cardiac surgery, including CABG and valve replacement, controversy remains regarding the 
safety of preoperative antiplatelet therapy. Aspirin's benefits of improving graft patency and preventing perioperative ischemic complications may be overcome by the risk of bleeding. For safety concern, aspirin is recommended to be withdrawn two to 10 days before elective cardiac surgery. Only for patients with acute coronary syndrome, preoperative aspirin with dosage of $100 \mathrm{mg}-325 \mathrm{mg}$ is recommended [Dunning 2008; Hillis 2011]. Besides the above concerns, some studies also found protective effect of preoperative aspirin on CSA-AKI [Hur 2017; Huang 2015; Yao 2015; Cao 2012]. Other studies found no positive effect of preoperative aspirin [Mazzeffi 2016; Myles 2016].

To summarize the literature and clarify the preventive effect of preoperative aspirin in CSA-AKI, we performed a meta-analysis of observational and randomized controlled trials (RCTs) that evaluated the efficacy of aspirin use in the preoperative period.

\section{METHODS}

Study search: MEDLINE, EMBASE, and the Cochrane Databases were searched in May 2019 for potentially eligible studies published in English. The search terms included acetylsalicylic acid, acetylsalicylate, aspirin, ASA, coronary artery bypass, CABG, cardiac surgery, valve replacement, acute kidney injury, acute kidney failure, acute renal injury, acute renal failure, and dialysis. The reference lists of relevant papers also were searched.

Eligibility and data extraction: Studies were eligible for inclusion if they met all of the following criteria: (i) RCTs or observational cohort studies; (ii) involving adult patients (age >18 years); (iii) undergoing CABG, valve replacement, or combined procedures; (iv) the presence of a group receiving aspirin preoperatively compared with a control group (aspirin discontinued or placebo given); and (v) offering incidence of post-operative acute kidney injury. Studies or subgroups of patients in which the aspirin or control group received other anticoagulants or antiplatelet drugs were excluded. Eligibility assessment and data extraction independently were performed in a blind and standardized fashion by two reviewers $(\mathrm{H}$. Liu and J. $\mathrm{Su}$ ). Extracted data included assessment of study quality, study design, patient demographics, baseline characteristics, aspirin dose, number of days free of aspirin prior to surgery (control group only), and safety and efficacy endpoints. Disagreements between reviewers were resolved by consensus.

Risk of bias assessment: The methodological quality of RCTs was assessed by the Jadad Scale. The methodological quality of cohort studies was assessed by the NewcastleOttawa Scale, which consists of three factors: patient selection, comparability of the study groups, and assessment of outcome. A score of 0 to 9 (allocated as stars) was allocated to each cohort study.

Data analysis: This meta-analysis was performed, according to the Preferred Reporting Items for Systematic Reviews and Meta-Analysis (PRISMA) statements.

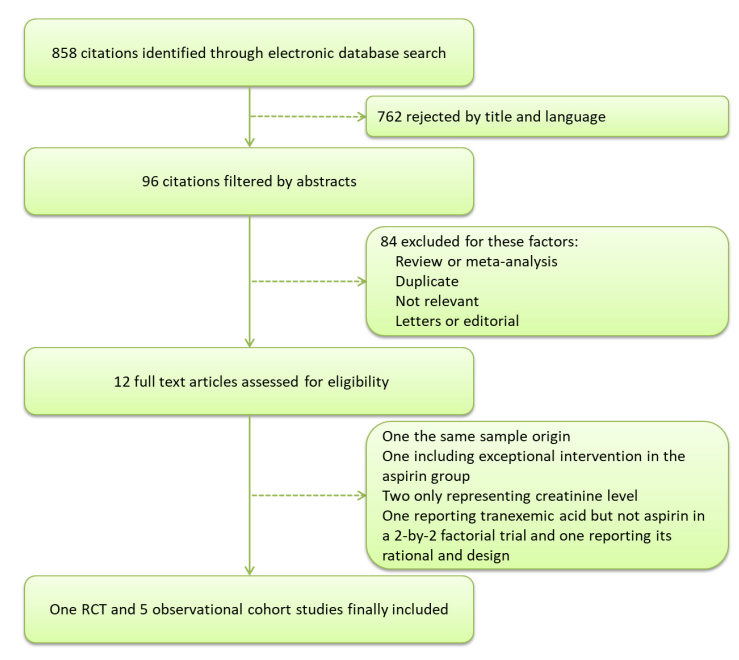

Figure 1. Attrition diagram for literature search

Dichotomous variables are expressed as odds ratio (OR) and $95 \%$ confidence interval (CI). The heterogeneity among trials was evaluated with the chi-square test. I2 statistic was used as a measure of percent of total variability, due to heterogeneity among studies. High heterogeneity was considered present with chi-square test $\mathrm{P}<0.10$ and/ or I $2 \geq 50 \%$. The random-effect model was used if there was high heterogeneity across trials. If not, the fixed-effect model with the Mantel-Haenszel method was performed. Treatment groups that discontinued aspirin less than seven days before surgery were analyzed in the preoperative aspirin group based on aspirin's ability to irreversibly inhibit platelet function for the life of the platelet (five to 10 days). Publication bias was assessed using funnel plots and the Egger's test for asymmetry. A P-value of $<0.1$ was considered to represent significant asymmetry. Sensitivity analyses (exclusion of one study at a time) were performed to determine the stability of the overall effects. All $\mathrm{P}$ values were two-tailed with the statistical significance set at $<0.05$. Statistical analyses were conducted using Review Manager 5.3 (Copenhagen: The Nordic Cochrane Centre, The Cochrane Collaboration, 2014).

\section{RESULTS}

Search results: From 858 initial citations, six eligible studies, which included a total of 11,406 patients, were identified in the present meta-analysis [Hur 2017; Mazzeffi 2016; Huang 2015; Yao 2015; Cao 2012; Myles 2016], with 6,280 patients in the aspirin group and 5,126 in the control group. Characteristics of the included studies and patients are summarized in Table 1. Four of the included studies defined the aspirin group as taking aspirin within five days before surgery, but did not mention the exact dosage (Table 1). The other two studies defined the aspirin group as taking aspirin within 24 hours before surgery with a low dose $(75 \mathrm{mg}-100 \mathrm{mg}$ 


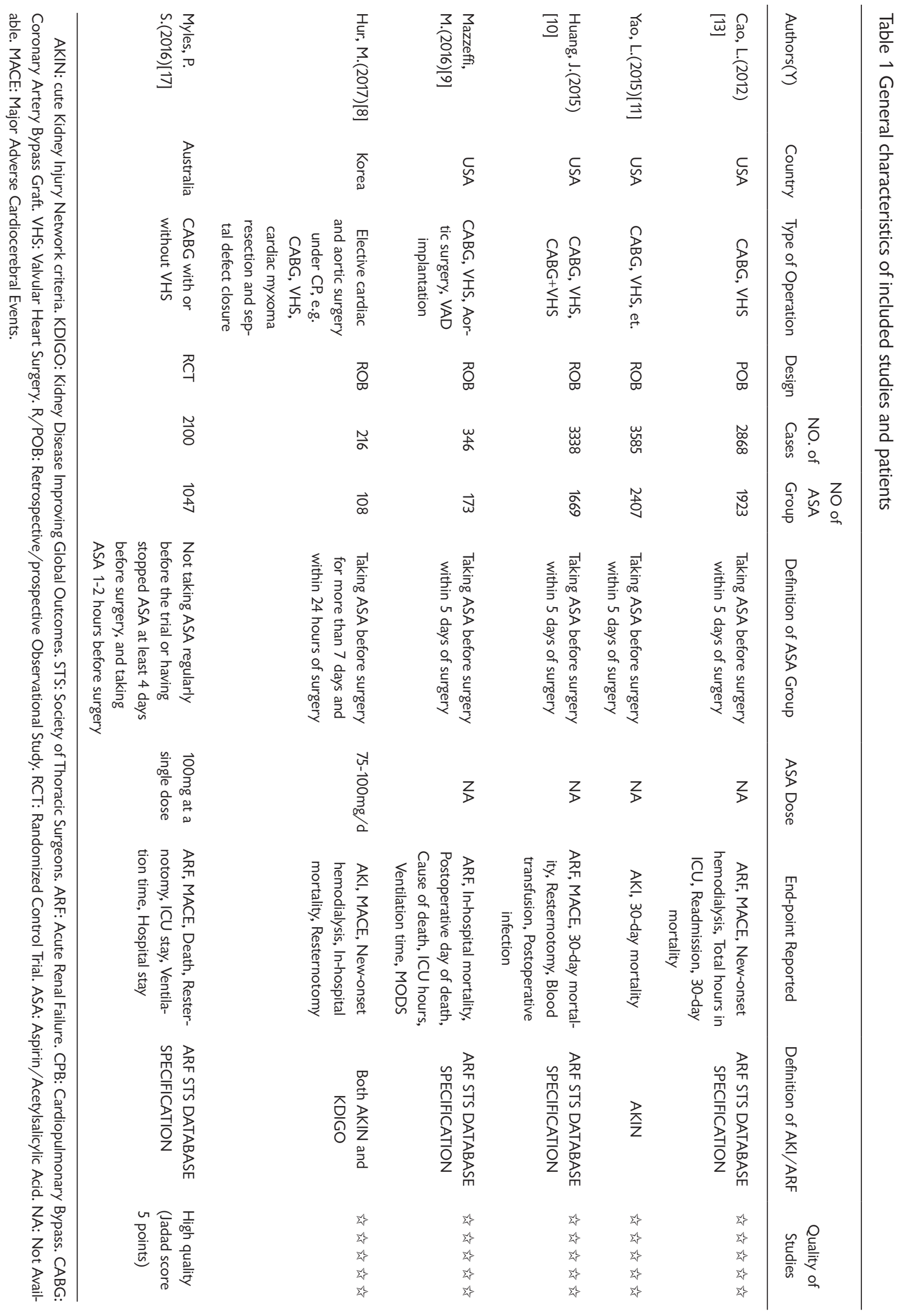




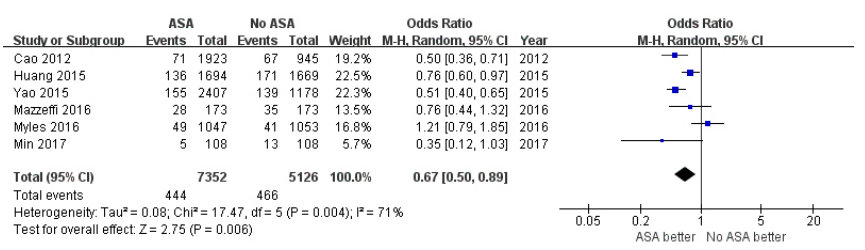

Figure 2. Analysis of post-operative acute renal failure in patients with preoperative aspirin versus control

per day or in a single dose). KDIGO, AKIN, or ARF STS Database Specification were applied to defined acute kidney injury or acute renal failure.

Quality of the studies: All studies eligible for the review were divided into randomized controlled trials and observational trials. Of these six studies, only Myles et al conducted a prospective randomized trial. The other five studies were prospective or retrospective observational studies and propensityscore matched. The RCT, according to Jadad score, was of high quality [Myles 2008]. The other five observational studies were assessed according to Newcastle-Ottawa Scale (NOS) and the score were all more than 6 points (Table 1).

Efficacy outcomes: Acute Renal Failure -- in the six included studies, Myles [Myles 2016] et al and Mazzeffi [Mazzeffi 2016] et al found no significant protective effect of aspirin on CSAAKI. Overall, preoperative aspirin significantly decreased the risk of CSA-AKI (OR, 0.67; 95\% CI, 0.50-0.89; $\mathrm{P}=0.006$ ) (Figure 2).

Safety outcomes: Major Adverse Cardiocerebral Events -- MACEs referred to a composite outcome. It included permanent or transient stroke, coma, perioperative myocardial infarction, heart block, and cardiac arrest, according to the STS national criteria. Four of six articles reported the incidence of a MACE with a total of 8,547 patients. Preoperative aspirin significantly decreased the risk of a MACE (OR, $0.88 ; 95 \%$ CI, 0.76-1.01; $\mathrm{P}=0.07$ ) (Figure 3). Another four of six articles specifically reported the rate of post-operative stroke with a total of 8,677 patients. Preoperative aspirin led to an insignificant decrease in post-operative stroke by $16 \%$ (OR, 0.84; 95\% CI, 0.63-1.11; P = 0.22) (Figure 4).

All-Cause Mortality: All six articles reported mortality. Hur et al [Hur 2017] and Mazzeffi et al [Mazzeffi 2016] reported in-hospital, all-cause mortality without time restriction. The other four studies reported a 30-day, allcause mortality. All-cause mortality significantly was lower in the aspirin group (OR, 0.64; 95\% CI, 0.53-0.77; $\mathrm{P}<$ 0.001) (Figure 5).

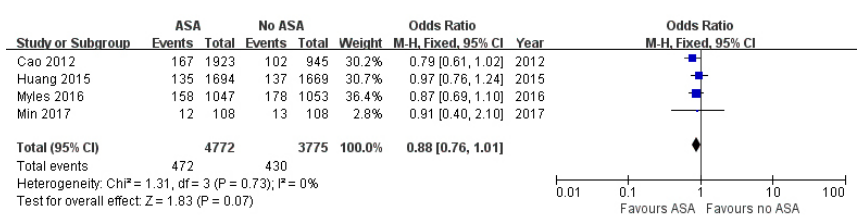

Figure 3. Analysis of post-operative MACEs in patients with pre-operative aspirin versus control

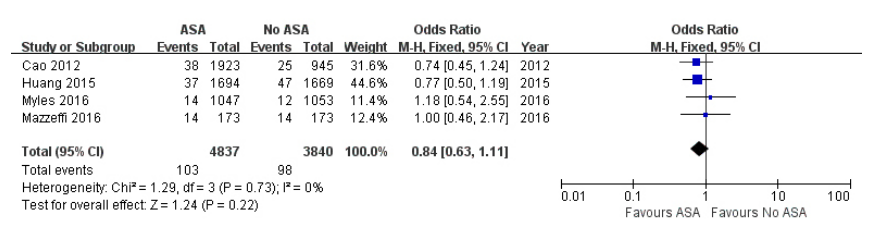

Figure 4. Analysis of post-operative stroke in patients with preoperative aspirin versus control

Resternotomy for Bleeding: Three articles reported the incidence of resternotomy for major bleeding in 5,679 patients. Preoperative aspirin did not show significant adverse impact on resternotomy (OR, 1.01; 95\% CI, 0.761.34; $\mathrm{P}=0.95)$ (Figure 6).

\section{DIsCussion}

The key finding of our meta-analysis is that preoperative aspirin lowers the risk of post-operative acute renal failure in patients who had undergone cardiac surgeries without increasing the rate of resternotomy for major bleeding. It also reduces risks of MACE and in-hospital mortality. Most of the studies were new and of high quality.

COX-1 catalyzes oxidation of arachidonic acid derived from membrane phospholipids. It also generates cyclic prostanoids. The latter includes TXA2 and prostacyclin, which are mainstream of inflammatory agents [Awtry 2000]. Aspirin irreversibly inhibits activity of COX-1. Inhibition of platelet TXA2 formation prevents aggregation and activation of platelets. This pharmacological effect is essential for aspirin's application in CAD [Fuster 1993]. Since platelets are anucleate with limited mRNA pool and protein synthesis, the inhibition of platelet function is also irreversible. And, a single dose of $100 \mathrm{mg}$ aspirin is enough for inhibition of platelet COX-1 in most individuals. However, inhibition of endothelial COX-1 may decrease prostaglandins production, resulting in platelet activation and vasoconstriction. But for endothelial cells, COX-1 can be regenerated, thus weakening the effect of aspirin on endothelial function. All in all, higher aspirin doses are no more effective in antagonizing platelet function. It can lead to strong endothelial COX-1 inhibition, which contrarily causes vasoconstriction and ischemia, compromising the antithrombotic effect. Higher aspirin doses also increase risks of bleeding and gastrointestinal side effects.

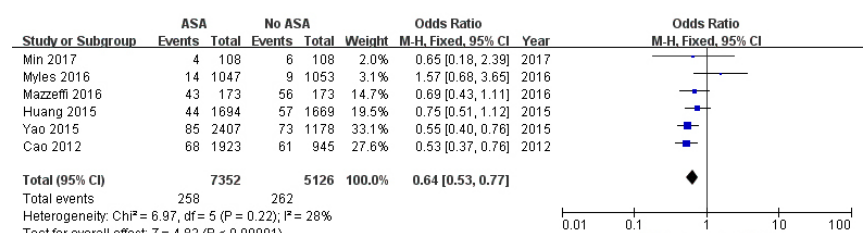

Figure 5. Analysis of all-cause mortality in patients with preoperative aspirin versus control 


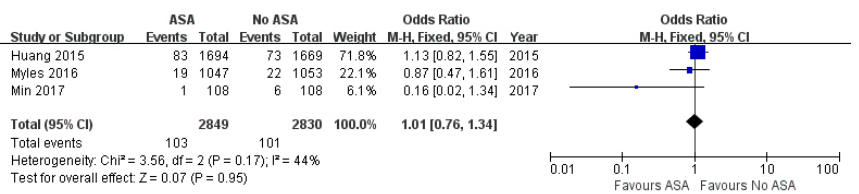

Figure 6. Analysis of resternotomy in patients with preoperative aspirin versus control

For CAD patients undergoing surgical intervention, aspirin strongly is recommended to be initiated early post-operatively to reduce the risk of saphenous vein graft closure and ischemic complications, including stroke, myocardial infarct, and renal failure [Hillis 2011; Mangano 2002]. But there is no consensus on a preoperative aspirin strategy. Concerning risk of major bleeding and prolonged wound closure, early guidelines recommend cessation of aspirin two to 10 days before elective cardiac surgery for platelet function turnover [Hur 2017]. Recent studies, however, found that preoperative aspirin might not increase the risk of bleeding [Xiao 2015]. Or, it might not increase the risk of reoperation for bleeding complications, even if it did increase blood loss or need for a red blood cell transfusion [Jacob 2011; Goldhammer 2015; Bybee 2005]. This is consistent with our finding. Systematic review by Sun et al [Sun 2008] further revealed that preoperative aspirin increased post-operative bleeding (without increasing risk of reoperation), but this might be avoided by lowering doses to less than $325 \mathrm{mg} /$ day. And low-dose preoperative aspirin is considered safe in patients undergoing CABG [Hillis 2011; Aboul-Hassan 2017].

If it's safe to continue low-dose aspirin before surgical procedure, then we come to question the efficacy of preoperative aspirin to protect against AKI and death. Our study suggested that preoperative low-dose aspirin prescribed five days preceding surgery decreased the occurrence of post-operative renal failure, a severe form of CSA-AKI. The occurrence of CSA-AKI mainly is caused by ischemicreperfusion injury especially due to systemic inflammatory reaction during extracorporeal circulation [Fuhrman 2017; Wang 2017]. In such pathophysiological context, current key principles for prevention of CSA-AKI are maintaining renal perfusion during surgical procedure and moderating inflammatory response in kidney, during surgery and after reperfusion [Wang 2017; Shin 2016]. It is thought that the activation of blood elements mediates the principle complications of CPB in the form of inflammatory system activation. They include leucocytes, complements, and platelets. Neutrophils are the main target of the whole body inflammatory response, during CPB [Yoshimura 2003; Nilsson 1988]. Up-regulation of adhesion molecules induces neutrophil sequestration in the organ capillaries. Platelet activation and platelet-leukocyte conjugate generate aggregates and microemboli. Together with sequestrated neutrophils, they impair whole blood filterability. Microaggregates of platelets and platelet-leucocyte may physically be sequestered in the renal vascular beds, impairing microcirculation while triggering local vasoactive changes and the inflammatory reaction.
From this standpoint, the mechanism of aspirin in protecting renal function may be explained by its ability to inhibit platelet function. It reduces microthrombi formation to maintain microcirculation, during operation and prevent post-operative non-reflow phenomenon. This presumption mainly is based on the traditional way of platelet function. Besides, platelets also may work in a nontraditional way to impair kidney circulation and function during CPB. Association of platelet with innate immunity and SIRS is under heated discussion nowadays. In a murine model of ARDS due to abdominal sepsis, platelet-depleted mice had reduced infiltration of neutrophils, less pulmonary edema formation and better outcomes, which may be secondary to the diminished leucocyte recruitment [Asaduzzaman 2009]. Now, it's recognized that there is a close evolutionary association between the inflammatory cascade and the hemostatic system, in which platelet activation is crucial [Toner 2015]. Platelets, though anucleate, carry certain precursor mRNAs for pro-inflammatory factors, such as IL-1 $\beta$. On activation, platelets synthesize IL-1 $\beta$. IL-1 $\beta$ and promotes the adherence of polymorphonuclear leukocytes to endothelium and enhances cell trafficking [Lindemann 2001]. Activated platelets also upregulate the expression of adhesion receptors and promote platelet adhesion with the endothelium and leucocytes. Inflammatory and thrombotic agents are synthesized and released, enhancing leucocyte recruitment, edema formation, and production of neutrophil extracellular traps [Smith 2011]. Edema of interstitial tissue partially is due to endothelium junction impairment. Microaggregates and platelet-leucocyte conjugates in the microcirculation, as well as edema of interstitial tissue, lead to no-reflow and irreversible renal injury after surgical procedure [Levi 2016]. Edema of renal interstitium also adds to the intracapsular pressure, decreases filtration gradient, and even causes "intracapsular tamponade" [Kashani 2017]. It further impedes glomerular filtration. But the mechanism of platelet-leukocyte interaction still is under debate. It is found that platelet-mediated recruitment of neutrophils are not solely induced by the activation of COX-2 and production of prostaglandins, but also by non-COX mechanisms in COX-2 deficient mice [Weissmann 2002].

Generally, platelets play a critical role in thrombosisrelated ischemia and ischemic-reperfusion injury induced by innate inflammatory responses. Aspirin, by impeding aspirin function, is beneficial in preventing CSA-AKI.

Aspirin also reduces all-cause mortality in our study. A prospective study of 1,636 patients suggested that taking aspirin within five days preceding surgery significantly lowers inhospital mortality (OR, 0.34; 95\% CI, 0.15-0.75; P = 0.007) [Bybee 2005]. Deng et al [Deng 2015] also found a dosage effect of preoperative aspirin in mortality, that 30-day allcause mortality significantly was lower in patients who took $81 \mathrm{mg}$ of aspirin 24 hours or less preoperatively (OR, 0.34; $95 \%$ CI, 0.18-0.66; $\mathrm{P}<0.01)$, but not for whom taken $325 \mathrm{mg}$ of aspirin (OR, 0.74; 95\% CI, 0.41-1.35; P = 0.33) compared with no aspirin.

Our meta-analysis also has limitations. First of all, there were variations in aspirin dosage and timing of prescription. Besides, most studies included were observational studies and 
inherently were biased for their non-randomized and nonblinded design. But the authors tried to eliminate selection bias by conducting propensity-score matching before data analysis. Since only one study [Huang 2015] has differentially analyzed efficacy of preoperative aspirin in either CABG or VHS, and no study compares on-pump and off-pump surgeries in detail, we could not recommend aspirin prescription in specific surgical settings. On the other hand, there were only six studies included in our meta-analysis. And publication bias would be difficult to evaluate. Also, heterogeneity among the studies should not be overlooked.

All in all, preoperative low-dose aspirin may be promising in preventing CSA-AKI without increasing the risk of bleeding. Continuing aspirin therapy also can help decrease peri-operative MACEs for patients with coronary or cerebrovascular atherosclerosis. We also suggest more randomized controlled trials be designed and carried out to clarify the following problems: 1) optimal strategy of pre-operative aspirin prescription as to the dosage, duration, and timing; 2) efficacy and safety issues in patients with different underlying diseases; 3) efficacy and safety issues respectively in on-pump and off-pump cardiac surgeries.

\section{CONCLUSION}

Preoperative low-dose aspirin decreases post-operative CSA-AKI, mortality, and MACEs without increasing risk of reexploration. But most of the studies are observational and lack a uniform standard on prescription of aspirin, outcomes measurement, and stratification analysis concerning different types of surgical procedures. More randomized controlled trials are necessary to confirm the efficacy and safety of preoperative aspirin prescription.

\section{REFERENCES}

Aboul-Hassan SS, Stankowski T, Marczak J, Peksa M, Nawotka M, Stanislawski R, Kryszkowski B, Cichon R. 2017. The use of preoperative aspirin in cardiac surgery: A systematic review and meta-analysis. J Card Surg 32(12):758-774.

Alshaikh HN, Katz NM, Gani F, Nagarajan N, Canner JK, Kacker S, Najjar PA, Higgins RS, Schneider EB. 2017. Financial Impact of Acute Kidney Injury After Cardiac Operations in the United States. ANN THORAC SURG.

Asaduzzaman M, Lavasani S, Rahman M, Zhang S, Braun OO, Jeppsson B, Thorlacius H. 2009. Platelets support pulmonary recruitment of neutrophils in abdominal sepsis. CRIT CARE MED 37(4):1389-1396.

Awtry EH, Loscalzo J. 2000. Aspirin. CIRCULATION 101(10):1206-1218.

Bybee KA, Powell BD, Valeti U, Rosales AG, Kopecky SL, Mullany C, Wright RS. 2005. Preoperative aspirin therapy is associated with improved postoperative outcomes in patients undergoing coronary artery bypass grafting. CIRCULATION 112(9 Suppl): I286-I292.

Cao L, Silvestry S, Zhao N, Diehl J, Sun J. 2012. Effects of preoperative aspirin on cardiocerebral and renal complications in non-emergent cardiac surgery patients: a sub-group and cohort study. PLOS ONE 7(2):e30094.
Cao L, Young N, Liu H, Silvestry S, Sun W, Zhao N, Diehl J, Sun J. 2012. Preoperative aspirin use and outcomes in cardiac surgery patients. ANN SURG 255(2):399-404.

Deng Y, Pisklak PV, Lee VV, Tolpin DA, Collard CD, Elayda MA, Coselli J, Pan W. 2015. Association Between Preoperative Aspirin-dosing Strategy and Mortality After Coronary Artery Bypass Graft Surgery. ANN SURG 262(6):1150-1156.

Dunning J, Versteegh M, Fabbri A, Pavie A, Kolh P, Lockowandt U, Nashef SA. 2008. Guideline on antiplatelet and anticoagulation management in cardiac surgery. Eur J Cardiothorac Surg 34(1):73-92.

Fuhrman DY, Kellum JA. 2017. Epidemiology and pathophysiology of cardiac surgery-associated acute kidney injury. Curr Opin Anaesthesiol 30(1):60-65.

Fuster V, Dyken ML, Vokonas PS, Hennekens C. 1993. Aspirin as a therapeutic agent in cardiovascular disease. Special Writing Group. CIRCULATION 87(2):659-675.

Goldhammer JE, Marhefka GD, Daskalakis C, Berguson MW, Bowen JE, Diehl JT, Sun J. 2015. The Effect of Aspirin on Bleeding and Transfusion in Contemporary Cardiac Surgery. PLOS ONE 10(7):e134670.

Hillis LD, Smith PK, Anderson JL, Bittl JA, Bridges CR, Byrne JG, Cigarroa JE, Disesa VJ, Hiratzka LF, Hutter AJ et al. 2011. 2011 ACCF/ AHA Guideline for Coronary Artery Bypass Graft Surgery: a report of the American College of Cardiology Foundation/American Heart Association Task Force on Practice Guidelines. CIRCULATION 124(23):e652-e735.

Hoste E, Vandenberghe W. 2017. Epidemiology of cardiac surgeryassociated acute kidney injury. Best Pract Res Clin Anaesthesiol 31(3):299-303.

Huang J, Donneyong M, Trivedi J, Barnard A, Chaney J, Dotson A, Raymer S, Cheng A, Liu H, Slaughter MS. 2015. Preoperative Aspirin Use and Its Effect on Adverse Events in Patients Undergoing Cardiac Operations. ANN THORAC SURG 99(6):1975-1981.

Hur M, Koo CH, Lee HC, Park SK, Kim M, Kim WH, Kim JT, Bahk JH. 2017. Preoperative aspirin use and acute kidney injury after cardiac surgery: A propensity-score matched observational study. PLOS ONE 12(5):e177201.

Jacob M, Smedira N, Blackstone E, Williams S, Cho L. 2011. Effect of timing of chronic preoperative aspirin discontinuation on morbidity and mortality in coronary artery bypass surgery. CIRCULATION 2011, 123(6):577-583.

Kashani KB, Mao SA, Safadi S, Amiot BP, Glorioso JM, Lieske JC, Nyberg SL, Zhang X. 2017. Association between kidney intracapsular pressure and ultrasound elastography. CRIT CARE 21(1):251.

Kulik A, Ruel M, Jneid H, Ferguson TB, Hiratzka LF, Ikonomidis JS, Lopez-Jimenez F, McNallan SM, Patel M, Roger VL et al. 2015. Secondary prevention after coronary artery bypass graft surgery: a scientific statement from the American Heart Association. CIRCULATION 131(10):927-964.

Levi Y, Sultan A, Alemayehu M, Wall S, Lavi S. 2016. Association of endothelial dysfunction and no-reflow during primary percutaneous coronary intervention for ST-elevation myocardial infarction. Cardiovasc Revasc Med 17(8):552-555.

Lindemann S, Tolley ND, Dixon DA, McIntyre TM, Prescott SM, Zimmerman GA, Weyrich AS. 2001. Activated platelets mediate inflammatory signaling by regulated interleukin 1 beta synthesis. J CELL BIOL 154(3):485-490. 
Mangano DT. 2002. Aspirin and mortality from coronary bypass surgery. N Engl J Med 347(17):1309-1317.

Mazzeffi M, Galvagno S, Gammie JS, Tanaka K. 2016. Impact of aspirin use on morbidity and mortality in massively transfused cardiac surgery patients: a propensity score matched cohort study. J ANESTH 30(5):817-825.

Myles PS, Smith JA, Forbes A, Silbert B, Jayarajah M, Painter T, Cooper DJ, Marasco S, McNeil J, Bussieres JS et al. 2016. Stopping vs. Continuing Aspirin before Coronary Artery Surgery. N Engl J Med 374(8):728-737.

Myles PS, Smith J, Knight J, Cooper DJ, Silbert B, McNeil J, Esmore DS, Buxton B, Krum H, Forbes A et al. 2008. Aspirin and Tranexamic Acid for Coronary Artery Surgery (ATACAS) Trial: rationale and design. AM HEART J 155(2):224-230.

Nilsson L, Brunnkvist S, Nilsson U, Nystrom SO, Tyden H, Venge P, Aberg T. 1988. Activation of inflammatory systems during cardiopulmonary bypass. Scand J Thorac Cardiovasc Surg 22(1):51-53.

Rosner MH, Okusa MD. 2006. Acute kidney injury associated with cardiac surgery. Clin J Am Soc Nephrol 1(1):19-32.

Shin SR, Kim WH, Kim DJ, Shin IW, Sohn JT. 2016. Prediction and Prevention of Acute Kidney Injury after Cardiac Surgery. BIOMED RES INT 2016:2985148.

Smith TL, Weyrich AS. 2011. Platelets as central mediators of systemic inflammatory responses. THROMB RES 127(5):391-394.

Sun JC, Whitlock R, Cheng J, Eikelboom JW, Thabane L, Crowther
MA, Teoh KH. 2008. The effect of pre-operative aspirin on bleeding, transfusion, myocardial infarction, and mortality in coronary artery bypass surgery: a systematic review of randomized and observational studies. EUR HEART J 29(8):1057-1071.

Toner P, McAuley DF, Shyamsundar M. 2015. Aspirin as a potential treatment in sepsis or acute respiratory distress syndrome. CRIT CARE 19:374.

Wang Y, Bellomo R. 2017. Cardiac surgery-associated acute kidney injury: risk factors, pathophysiology and treatment. NAT REV NEPHROL 13(11):697-711.

Weissmann G, Montesinos MC, Pillinger M, Cronstein BN. 2002. Nonprostaglandin effects of aspirin III and salicylate: inhibition of integrindependent human neutrophil aggregation and inflammation in COX 2- and NF kappa B (P105)-knockout mice. ADV EXP MED BIOL 507:571-577.

Xiao F, Wu H, Sun H, Pan S, Xu J, Song Y. 2015. Effect of preoperatively continued aspirin use on early and mid-term outcomes in off-pump coronary bypass surgery: a propensity score-matched study of 1418 patients. PLOS ONE 10(2):e116311.

Yao L, Young N, Liu H, Li Z, Sun W, Goldhammer J, Tao L, He J, Diehl J, Sun J. 2015. Evidence for preoperative aspirin improving major outcomes in patients with chronic kidney disease undergoing cardiac surgery: a cohort study. ANN SURG 261(1):207-212.

Yoshimura Y, Hiramatsu Y, Sato Y, Homma S, Enomoto Y, Kikuchi Y, Sakakibara Y. 2003. Activated neutrophils and platelet microaggregates impede blood filterability through microchannels during simulated extracorporeal circulation. ANN THORAC SURG 75(4):1254-1260. 